\title{
ISARC-2008
}

\section{DECISION SUPPORT MODEL USING THE ADABOOST ALGORITHM TO SELECT FORMWORK SYSTEMS IN HIGH-RISE BUILDING CONSTRUCTION}

\author{
Yoonseok Shin \\ Ph.D. Candidate \\ Department of Civil, Environmental and \\ Architectural Engineering, Korea University, \\ Anam-Dong, Seongbuk-gu, Seoul 136-713, \\ Republic of Korea \\ yoonseok@korea.ac.kr

\section{Sung-Woo Yang} \\ Graduate student \\ Department of Civil, Environmental and \\ Architectural Engineering, Korea University, \\ Anam-Dong, Seongbuk-gu, Seoul 136-713, \\ Republic of Korea \\ tearsi23@korea.ac.kr
}

\author{
Dae-Won Kim \\ Ph.D. Candidate \\ Department of Civil, Environmental and \\ Architectural Engineering, Korea University, \\ Anam-Dong, Seongbuk-gu, Seoul 136-713, \\ Republic of Korea \\ mtmkdw@korea.ac.kr

\section{Hun-Hee Cho} \\ Assistant Professor \\ Department of Civil, Environmental and \\ Architectural Engineering, Korea University, \\ Anam-Dong, Seongbuk-gu, Seoul 136-713, \\ Republic of Korea \\ hhcho@korea.ac.kr
}

\section{Kyung-In Kang}

Professor

Department of Civil, Environmental and Architectural Engineering, Korea University, Anam-Dong, Seongbuk-gu, Seoul 136-713, Republic of Korea

kikang@korea.ac.kr

\begin{abstract}
Appropriate selection of a formwork system is a crucial factor in successfully completing any high-rise building construction project. However, in practice, the selection of an appropriate formwork system has depended mainly on the intuitive and subjective opinion of practitioners with restricted experience. Therefore, in this study, a decision support model using AdaBoost is proposed to select a formwork system suitable for the construction site conditions. To validate the proposed model, the selection models AdaBoost and ANN were both applied to actual case data of high-rise building construction in Korea. The AdaBoost model showed slightly better accuracy than that of the ANN model. The AdaBoost model can assist engineers to determine the appropriate formwork system at the inception of future projects.
\end{abstract}




\section{KEYWORDS}

formwork system, adaptive boosting (AdaBoost), artificial neural network (ANN), decision support model, high-rise building

\section{INTRODUCTION}

In high-rise building construction, appropriate selection of the formwork system is a crucial factor for successful project completion with reinforced concrete (RC) structures. Formwork operations account for a large portion of the cost of the building structure, representing $40 \sim 60 \%$ of the cost of the concrete framework and $10 \%$ of the total building cost [1]. Moreover, formwork has an important role in the cycle times per floor in the building construction project. Therefore, the selection of an appropriate formwork system not only affects the entire construction duration and cost, but also affects subsequent construction activities such as electrical, mechanical, and finishing work [2]. However, in practice, the selection of the appropriate formwork system has depended mainly on the subjective and intuitive opinions of practitioners with restricted experience, even though construction methods have diversified as technologies have improved [2].

Artificial intelligence (AI) techniques have been used to assist in selecting formwork systems. Expert systems [3] and Artificial Neural Networks (ANNs) $[2,4]$ have been used because of the complexity of selecting formwork systems, which is influenced by various factors. Expert systems are a popular technique in construction engineering and management, but they have disadvantages such as the lack of self-learning and a time-consuming rule acquisition process [5]. ANNs are good at function approximation, forecasting, classification, and optimization tasks. However, this approach is a black-box technique and many neural network algorithms require large amounts of training data and training cycles because the determination of various parameters associated with training algorithms is not straightforward [6].

Among recent AI theories, the AdaBoost (Adaptive Boosting) algorithm, which was introduced by Freund and Schapire (1999) [7], has become an important tool in machine learning and predicting models. AdaBoost provides an effective learning algorithm and strong bounds on generalization performance $[8,9]$. In classification problems, AdaBoost is fast, simple and easy to program, and has no parameters to tune (except for the number of rounds $\boldsymbol{T}$ ) [7-9]. Although AdaBoost has been actively utilized in other domains with these advantages, AdaBoost has not yet been studied for selecting formwork systems in the construction field.

Therefore, we propose a decision support model using AdaBoost to select a formwork system that will be suitable for construction site conditions. In the next section, current techniques for selecting formwork systems in Korea are reviewed. Section 3 briefly describes the AdaBoost algorithm. The case study performed to verify the AdaBoost model for selecting formwork systems by applying it to real case data is described in Section 4. Section 5 shows the results and discussion of the application. Finally, conclusions and suggestions for further studies are presented in Section 6.

\section{SELECTION OF FORMWORK SYSTEMS IN KOREA}

Recent developments in formwork technologies have resulted in a number of highly specialized formwork systems that are already in use. Kim's study in 2007 [10] showed that there are main five types of horizontal formwork systems used in 61 construction sites in Korea, as shown in Figure 1. In recent years, aluminum forms have been most preferred by contractors. Formwork in Korea remains strongly labor intensive.

The process of selecting a formwork system is shown in Figure 2 [11]. In this process, the general contractor's planning engineers encounter the problem of having to choose from among various formwork systems, considering many factors relating to the site. It is very difficult to select an appropriate formwork system, even for very experienced practitioners. It is therefore necessary to support their decision making for proper formwork system selection. 


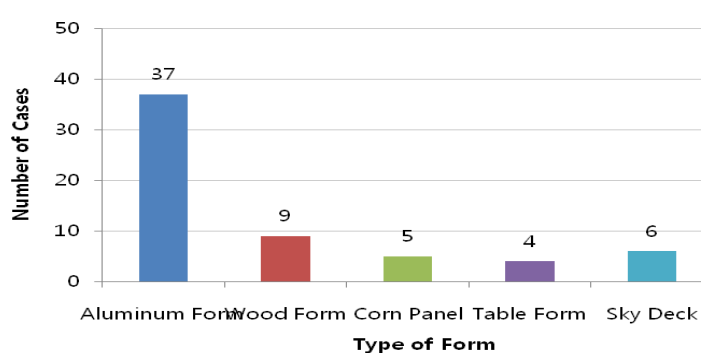

Figure 1. Horizontal Formwork Systems in Korea



Figure 2. Formwork Selection Process

There have been many studies of supporting the engineer making decisions on selecting a formwork system using AI techniques, such as expert systems and ANN. However, AdaBoost has seldom been introduced in the construction domain, although it has been actively utilized in classification problems in other domains, such as face detection, recognition of character, bankruptcy forecasting, credit scoring and so on. Therefore, this study applies AdaBoost and examines its applicability in selecting a formwork system in the construction domain.

\section{ADABOOST}

AdaBoost, which is introduced by Freund and Shapire in 1995 [12], makes maximum use of a classifier by improving its accuracy. It is a simple learning algorithm that builds a strong classifier from a small set of efficient but weak classifiers, as shown in Figure 3. The idea is to choose the weak classifiers in such a way that when combined they perform much better. In the result, the final strong classifier builds a model that is able to predict the class of a new observation given a data set.

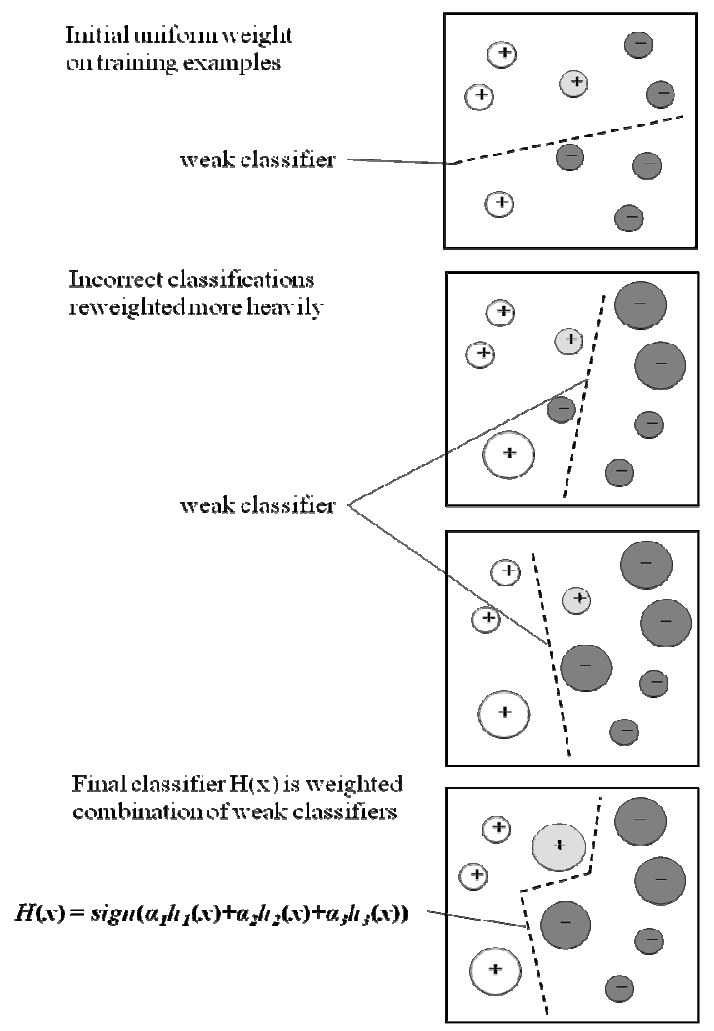

Figure 3. A Simple Example Showing how AdaBoost Constructs a Strong Classifier from a Set of Weak Classifiers

AdaBoost in this study is much the same as the boosting algorithm proposed by Viola and Jones (2001) [13]. In their algorithm, AdaBoost was used to select features and to train the classifier. AdaBoost can be used to boost the classification performance of a simple learning algorithm by combining collections of weak classifiers to form a stronger classifier. In the language of boosting algorithms, the simple learning algorithm is called a weak learner, which selects a small, efficient set of weak classifiers with the lowest classification error from a large number of potential features. The weak learner does not classify the training data well with 
even the best classification function. To boost the weak learner, it is called on to solve a sequence of learning problems. After the first round of learning, the examples are reweighted to emphasize those that were incorrectly classified by the previous weak classifier. The final strong classifier takes a weighted combination of the weak classifiers which determine the optimal threshold classification function for each feature. The algorithm for AdaBoost is shown in Figure 4 [13].

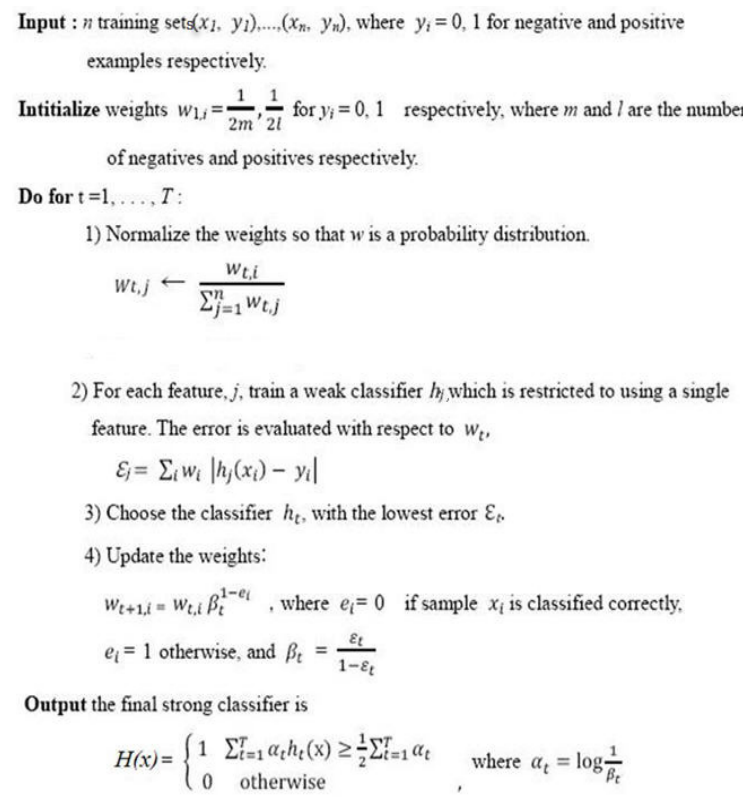

Figure 4. AdaBoost Algorithm (Viola and Jones, 2001)

\section{APPLICATION}

\subsection{Data Collection}

In this study, factors for selecting a horizontal formwork system were determined by reviewing the previous research $[1,14]$ and interviewing engineers experienced in formwork in Korea. As a result, 6 factors for selecting the slab formwork system were determined, and are shown in Table 1. In the algorithm in Figure 4, each of the site conditions, such as number of floors, area of typical floor, height of typical floor, and structural type, belongs to an $x_{i}$ of the training set, and the result showing whether the input data are the target (i.e., the right formwork system) or not is labelled $y_{i}$.
Data for 61 cases from 11 major general contractors were collected from high-rise building construction projects in Korea. Projects were limited to RC residential buildings that were higher than 30 stories. The result of selecting the formwork system was representatively classified into four form types: wood form, aluminum form, sky deck, and table form, which were generally used in these sites. The 61 cases were randomly divided into 41 training sets and 20 test sets.

Table 1. Variables for Input and Output

\begin{tabular}{|c|c|c|c|}
\hline $\begin{array}{c}\text { In- } \\
\text { put }\end{array}$ & $\begin{array}{c}\text { Typical } \\
\text { number of } \\
\text { floors }\end{array}$ & $\begin{array}{c}\text { Numeric } \\
\text { (Stories) }\end{array}$ & $\begin{array}{c}\text { Max. }=52, \\
\text { Min. }=30\end{array}$ \\
\hline $\begin{array}{c}\text { Typical } \\
\text { height of } \\
\text { floor }\end{array}$ & $\begin{array}{c}\text { Numeric } \\
(\mathrm{m})\end{array}$ & $\begin{array}{c}\text { Max. }=3.7, \\
\text { Min. }=2.6\end{array}$ \\
\hline $\begin{array}{c}\text { Typical } \\
\text { area of } \\
\text { floor }\end{array}$ & $\begin{array}{c}\text { Numeric } \\
\left(\mathrm{m}^{2}\right)\end{array}$ & $\begin{array}{c}\text { Max. }=3342, \\
\text { Min. }=347\end{array}$ \\
\hline $\begin{array}{c}\text { Average } \\
\text { cycle time } \\
\text { per floor }\end{array}$ & $\begin{array}{c}\text { Numeric } \\
(\text { day })\end{array}$ & $\begin{array}{c}\text { Max. }=8, \\
\text { Min. }=3\end{array}$ \\
\hline $\begin{array}{c}\text { Floor plan } \\
\text { shape }\end{array}$ & Nominal & $\begin{array}{c}\text { Tetragonal }=1, \\
\text { Others }=2\end{array}$ \\
\hline $\begin{array}{c}\text { Structural } \\
\text { type }\end{array}$ & Nominal & $\begin{array}{c}\text { Wall type }=1, \\
\text { Rigid frame }=2, \\
\text { Flat plate slab }=3\end{array}$ \\
\hline put & $\begin{array}{c}\text { Horizontal } \\
\text { form }\end{array}$ & Nominal & $\begin{array}{c}\text { Wood form }=1, \\
\text { Aluminum form }= \\
\text { 2, Sky deck }=3, \\
\text { Table form }=4\end{array}$ \\
\hline
\end{tabular}

\subsection{Applying AdaBoost to Selecting a Formwork System}

The AdaBoost model for selecting formwork systems was tested by applying it to real cases of high-rise building projects. AdaBoost is generally used with two-class problems with outcomes such as win or lose. The genuine AdaBoost therefore required some modification to be extended to multiclass cases. In this study, the strong classifiers for each formwork system were combined for multiclassification by computing an AdaBoost model using the $\mathrm{C}++$ language. In the model, one 
formwork system which gets the highest score from each strong classifier is recommended.

To verify the performance of the proposed model, the same cases were applied to the ANN model to compare the results. NeuroShell 2 Release 4.0 was used to develop the NN models in this study. To construct models with either AdaBoost or ANN, optimal parameters must be determined beforehand: the number of weak classifiers $(T)$ for AdaBoost, and the number of hidden neurons, the momentum, and a learning rate for ANN. We determined these values from repeated experiments. To evaluate the performance of both models for formwork system selection, fivefold cross-validation was performed.

\section{RESULTS AND DISCUSSION}

The results from 20 test data sets using AdaBoost and ANN are summarized in Table 2. The AdaBoost model's overall average accuracy was $76.8 \%$, with a minimum of $74 \%$ and a maximum of $79 \%$. When the number of weak classifiers $(T)$ was 10 , the accuracy was highest at $79 \%$. In this study, alterations of the $T$ value affected the average accuracy. The ANN model showed an average accuracy of $69 \%$ with the same data sets.

Table 2. Comparison of Accuracy of AdaBoost and ANN

\begin{tabular}{|c|c|c|c|c|c|c|}
\hline \multirow{2}{*}{ Experiment } & \multirow{5}{*}{ ANN } & \multicolumn{5}{|c|}{$\begin{array}{c}\text { AdaBoost (\%) } \\
\text { (Tumber of weak } \\
\text { classifiers) }\end{array}$} \\
\cline { 3 - 7 } & & 5 & 10 & 15 & 20 & 30 \\
\hline Fold 1 & 60 & 75 & 80 & 75 & 75 & 75 \\
\hline Fold 2 & 70 & 75 & 80 & 80 & 75 & 75 \\
\hline Fold 3 & 75 & 75 & 80 & 70 & 75 & 70 \\
\hline Fold 4 & 65 & 80 & 75 & 70 & 75 & 80 \\
\hline Fold 5 & 75 & 85 & 80 & 75 & 80 & 85 \\
\hline $\begin{array}{c}\text { Mean of } \\
\text { accuracy }\end{array}$ & 69 & 78 & 79 & 74 & 76 & 77 \\
\hline
\end{tabular}

The results show that the AdaBoost model is more accurate than the ANN model for this task. However, the gap of accuracies between the two models is very slight. It was therefore difficult to conclude that the AdaBoost model is better than the ANN model in selecting formwork systems. The main advantage of AdaBoost is that it was simple and easy to construct compared with the ANN models, because AdaBoost has only one parameter $(T)$.

These results reveal that the AdaBoost algorithm, which is a new AI approach in construction, is potentially useful for formwork system selection. The decision support model using AdaBoost developed in this study can assist engineers to avoid serious mistakes in selecting an optimum formwork system in the inception of a high-rise building project.

\section{CONCLUSION}

We have proposed a decision support model using the AdaBoost algorithm to select formwork systems that will be suitable for construction site conditions. To verify the performance of the proposed model, the study compared two different learning algorithms, AdaBoost and ANN, which are both attracting attention with high performance in various classification problems. In both models, actual case data of horizontal formwork systems in high-rise building construction in Korea were used for fivefold cross-validation. Both models gave good results in the selection of a formwork system. With its single parameter, AdaBoost was easier to use in constructing the model than ANN. AdaBoost models can assist engineers to determine the appropriate formwork system at the early stage of future projects.

In this study, only the basic applicability of AdaBoost has been examined. Therefore, detailed further studies, such as statistical verification for the factors considered in selecting formwork systems, is necessary for practical application.

\section{ACKNOWLEDGMENT}

This research was supported by a grant (code 03 R\&D C04-01) from Construction Core-Technology Research \& Development Program funded by Ministry of Construction \& Transportation of Korean government. The writer thanks Sung-Uk Jung, researcher in the Electronics and Telecommunications Research Institute (ETRI), for his help and valuable assistance in programming the AdaBoost algorithm for this article. 


\section{REFERENCES}

[1] Hanna, A. S. (2005) Concrete Formwork Systems, Marcel Dekker, Inc., New York.

[2] Tam, C. M., Tong, T. K. L., Lau, T. C. T. \& Chan, K. K. (2005) Selection of vertical formwork system by probabilistic neural networks models, Construction Management and Economics, Vol. 23, 245-254.

[3] Hanna, A. S., Willenbrock, J. H. \& Sanvido, V. E. (1992) Knowledge acquisition and development for formwork selection system, Journal of Construction Engineering and Management, Vol. 118, No. 1, 179-198.

[4] Kamarthi, S. V., Sanvido, V. E. \& Kumara, S. R. T. (1992) NEUROFORM-Neural network system for vertical formwork selection, Journal of Computing in Civil Engineering, Vol. 6, No. 2, 178-199.

[5] An, S. H., Park, U. Y., Kang, K. I., Cho, M. Y. \& Cho, H. H. (2007) Application of Support Vector Machines in assessing conceptual cost estimates, Journal of Computing in Civil Engineering, Vol. 21, No. 4, 259-264.

[6] Kumar, P. R. \& Ravi, V. (2007) Bankruptcy prediction in banks and firms via statistical and intelligent techniques - A review, European Journal of Operational Research, Vol. 180, 1-28.

[7] Freund, Y. \& Schapire, R. E. (1999) A short introduction to boosting, Journal of Japanese Society for Artificial Intelligence, Vol. 14, No. 5, 771-780.
[8] Papageorgiou, C., Oren, M. \& Poggio, T. (1998) A General Framework for Object Detection, Proceedings of International Conference on Computer Vision, 555-562.

[9] Schapire, R. E., Freund, Y., Bartlett, P. \& Lee, W. S. (1998) Boosting the margin: a new explanation for the effectiveness of voting methods, Ann. Stat. Vol. 26, No. 5, 1651-1686.

[10] Kim, T. H. (2007) Optimization of the Formwork Selection Process in Tall Buildings, MS Thesis, Korea University, Korea.

[11] Kim, D. E. (2006) Decision Support Models for Selection of Formwork Systems, MS Thesis, Korea University, Seoul, Korea.

[12] Freund, Y. \& Schapire, R. E. (1999) A short introduction to boosting, Journal of Japanese Society for Artificial Intelligence, Vol. 14, No. 5, 771-780.

[13] Viola, P. \& Jones, M. (2001) Rapid Object Detection using a Boosted Cascade of Simple Features, Proceedings of IEEE International Conference on Computer Vision and Pattern Recognition, 511-518.

[14] Proverb, D. G., Holt, G. D. \& Olomolaiye, P. O. (1999) Factors in formwork selection: a comparative investigation, Building Research \& Information, Vol. 27, No. 2, 109-119. 